\title{
Financiamiento de Generadoras Eléctricas de Energía Renovable en Perú
}

Gonzalo Soriano Castillo

Abogado por la Universidad de Lima. Miembro Asociado de ADV. Editores - Revista ADVOCATUS.

Profesor de Derecho Comercial de la Universidad de Lima. Máster en Derecho (LLM) en Energy Law and Policy por the University of Dundee.

SUMARIO:

I. Introducción.

II. La energía renovable en el Perú.

III. Project Finance: estructura más utilizada para financiamiento de energía renovable.

IV. Conclusiones y apuntes finales.

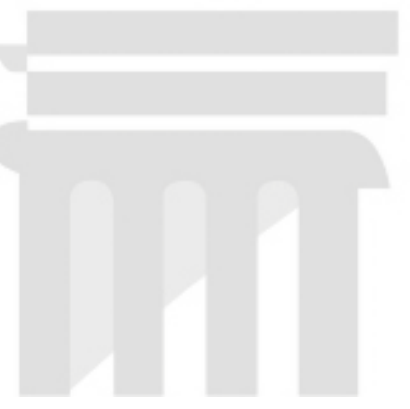




\title{
RESUMEN:
}

En el Perú, como parte de una tendencia global, desde hace más de una década, se viene promoviendo desde el Estado el desarrollo de generadoras de energías renovables bajo la firma de contratos Recursos Energéticos Renovables. En este artículo se ahondaremos en la metodología de financiamiento a la que acceden este tipo de proyectos estableciendo sus principales características y problemática.

Palabras Clave: Energía Renovable, Impacto Ambiental, Financiación de Proyectos, Tecnología, Mercado, Subsidios.

\begin{abstract}
:
In Peru, as part of a global trend, for more than a decade the development of renewable energy generators has been promoted from the State under the signing of RER contracts. In this article we will delve into the financing methodology to which this type of project has access, establishing its main characteristics and problems.
\end{abstract}

Keywords: Renewable Energy, Environmental Impact, Project Finance, Technology, Market, Subsidies.

\section{INTRODUCCIÓN}

Se entiende como energía renovable a aquella que se genera de fuentes naturales casi inagotables, ya sea por la incalculable cantidad de energía que estas contienen o por ser capaces de autogenerase por medios naturales.

Tomando en cuenta el desarrollo tecnológico y a su nivel de penetración en la matriz energética de cada país, a este tipo de energía se le clasifica en dos grandes ramas: (i) energías renovables convencionales y (ii) energías renovables no convencionales. Dentro de las primeras se encuentran las grandes centrales hidroeléctricas; mientras que dentro de las segundas se ubica a las pequeñas hidroeléctricas, las de generación eólica, la generación mediante energía solar, aquellas cuya matriz energética es fotovoltaica, entre otras.

Las energías renovables tienen como una de sus características más destacadas el ser fuente de energía limpia y crecientemente competitiva en cuanto a sus costos - aunque aun muchas veces más cara que el resto de energías-, ello en un claro contraste con la energía producida a partir de los combustibles fósiles.

Así, la principal diferencia con los combustibles fósiles radica en su abundancia y potencial de aprovechamiento en virtualmente todo el planeta, pero sobre todo en que no contribuyen al cambio climático mediante la producción de gases de efecto invernadero o emisiones contaminantes.

El crecimiento de las energías limpias en la matriz energética de los países es implacable e indetenible, como en el 2015 mostró la Agencia Internacional de la Energía ${ }^{1}$, estas representan cerca de la mitad de la nueva capacidad de generación eléctrica instalada en 2014 , constituyéndose conjuntamente en la segunda fuente global de electricidad, sólo superada por el carbón, aún predominante en China pero con una clara tendencia a su sostenida reducción.

De acuerdo también con dicha entidad, para mediados del presente siglo, la demanda mundial de electricidad aumentará en cerca de $70 \%$, elevando las energías renovables su participación de $18 \%$ al $24 \%$ en el mismo periodo- estimulada principalmente en regiones y países emergentes tales como India, China, Oriente Medio y África.

La implementación y mejora tecnológica de las energías limpias es fundamental para la lucha en la que debe abocarse el planeta contra el cambio climático y limitar sus efectos más de-

1. OIE/PNUD/ONUDI, 2010. 
vastadores. Conforme indica la Organización Meteorológica Mundial, adscrita a la Organización de las Naciones Unidas- en adelante, "ONU" - el periodo entre los años 2013 y 2017 fue considerado como el más caluroso jamás registrado, siendo el año 2017 el año más caluroso sin un evento de Niño y el 2016, el más caluroso de la historia².

Señala el mismo informe que la temperatura media mundial en 2017 fue superior en aproximadamente $0,46^{\circ} \mathrm{C}$ a la media a largo plazo del período $1981-2010-14,3^{\circ} \mathrm{C}$ - aunque eso solo muestra una parte de la historia. En paralelo a los aumentos de temperaturas, y como otra cara de la misma moneda, en el 2017 se produjeron fenómenos meteorológicos extremos en muchos países del mundo.

En dicho año, Estados Unidos tuvo que hacer frente al año más costoso de su historia en términos de desastres meteorológicos y climáticos. Según informó la Administración Oceánica y Atmosférica - NOAA - debido a una serie de incendios y huracanes el país afrontó pérdidas estimadas de 306000 millones de dólares, siendo el anterior el año 2005, en donde las perdidas bordearon los 215000 millones de dólares ${ }^{3}$.

Esta situación tiene como corolario además que uno de los objetivos establecidos por la ONU es lograr el acceso universal a la electricidad en $2030^{4}$, una meta quizás inalcanzable si consideramos que de seguir la tendencia actual la misma entidad calcula que habrá en esa fecha 800 millones de personas sin acceso real a electricidad.

Así, en este escenario, el estado peruano se embarcó en la tarea de promover proyectos de energía renovable, de energía limpia, y por promover nos referimos a volver comercialmente -y por ende bancable - el desarrollo de centrales de generación eléctrica de este tipo.

\section{LA ENERGÍA RENOVABLE EN PERÚ}

Perú ha sido tradicionalmente un país cuya matriz energética se ha sustentado en el sector hidroeléctrico, vale decir, en fuentes renovables. Esto significa que venimos contribuyendo sostenidamente a la reducción del efecto invernadero, y por ende al calentamiento global, que hoy agobia al planeta.

Según estadísticas del Organismo Supervisor de la Inversión en Energía y Minería - en adelante, "OSINERGMIN"-, en el año 2002, la electricidad generada por vías hidroeléctricas representó el $85 \%$ del total de energía generada en el país. Sin embargo, descubiertas y explotadas nuestras conocidas reservas gasíferas, con el proyecto de Camisea la participación de las hidroeléctricas disminuyó su porcentaje de participación hasta llegar al 61\% en el año 2008.

Es en ello que en mayo de 2008, el Estado Peruano emitió el Decreto Legislativo 1002, Decreto Legislativo de promoción de la inversión para la generación de electricidad con el uso de energías renovables —en adelante, "RER"—, tales como la energía solar, geotérmica, eólica, mareomotriz, la biomasa y las hidroeléctricas pequeñas con una capacidad instalada de hasta $20 \mathrm{MW}$. Este reglamento fue complementado luego, en marzo de 2011, por el nuevo Reglamento de la Generación de Electricidad con energías Renovables emitido en marzo de 2011 mediante Decreto Supremo Nº 012-2011-EM.

Según indica la normativa vigente, el fomento de las energías renovables, eliminando cual-

2. La Organización Meteorológica Mundial confirma 2017 como uno de los tres años más cálidos de los que se tienen datos. Enero 2018. Disponible en: <https://public.wmo.int/es/media/comunicados-de-prensa/laorganizaci\%C3\%B3n-meteorol\%C3\%B3gica-mundial-confirma-2017-como-uno-de-los-tres>.

3. Assessing the U.S. Climate in 2017. Disponible en: <https://www.ncei.noaa.gov/news/national-climate-201712>.

4. La ONU y el Banco Mundial impulsan el acceso universal a energía limpia y sostenible. Noviembre 2013. Disponible en: <http://www.un.org/content/es/ vidout/video1097.shtml>. 
quier barrera u obstáculo para su desarrollo, implica fomentar la diversificación de la matriz energética, constituyendo un avance hacia una política de seguridad energética y de protección del medio ambiente, siendo de interés público dar un marco legal en el cual se desarrollen estas energías que alienten estas inversiones y modifique las normas vigentes que no han sido efectivas al carecer de alicientes mínimos previstos en la legislación comparada.

Asimismo señala que la iniciativa normativa contempla beneficios adicionales tales como la implementación de un marco de fomento de la inversión privada, eliminando barreras, la preservación del medio ambiente con la producción de energías limpias, contribuyendo a lograr efectos positivos a nivel global $y$, al mismo tiempo, alcanzar una condición mínima de desarrollo de la economía peruana, la cual necesita una mayor seguridad en la disponibilidad de energía.

Bajo ese marco inicial y bajo sus posteriores modificaciones es que se han dado ya cuatro subastas RER en el país en la cual, en síntesis, el Estado invita a los postores a ofrecer venderle cierta capacidad de generación obteniendo la buena pro aquellos que puedan brindarla al menos precio por $\mathrm{Mwh}^{5}$.

En el cuadro siguiente puede apreciarse la oferta requerida en cada una de las cuatro subastas que hasta la fecha han ocurrido:
En el cuadro siguiente, se presentan los valores de las ofertas adjudicadas en las todas subastas con un desagregado en las tecnologías renovables de generación eléctrica. Se desprende que los precios han ido en caída libre, la reducción de los precios de las tecnologías RER a lo largo de las cuatro subastas realizadas es más que evidente.

El Perú posee experiencia en progreso en cuanto al financiamiento de inversión en energía renovable que cuente con el respaldo de apoyo estatal bajo el marco RER's, la cual sin duda es piedra angular para que la banca local, incluyendo a instituciones financieras como Scotiabank, BCP, BBVA Banco Continental e Interbank, se involucren en estas nuevas oportunidades de inversión.

Simultáneamente, entidades multilaterales o banca de fomento tales como CAF, IFC, BID, Kreditanstalt für Wiederaufbau - KFW - han puesto su experiencia regional a disposición de los promotores y del Estado peruano para afinar cada vez más los procesos subastas y las estructuras de financiamiento, las cuales van de la mano como veremos a continuación.

\section{PROJECT FINANCE: ESTRUCTURA MÁS UTILIZADA PARA FINANCIAMIENTO DE ENERGÍA RENOVABLE}

El financiamiento de energía renovable y en general cualquier método o alternativa para

\begin{tabular}{|c|c|c|c|c|}
\hline Requerimientos Subasta RER & $1^{\text {ra }}$ Subasta & $2^{\text {da }}$ Subasta & $3^{\text {ra }}$ Subasta & $4^{\text {ta }}$ Subasta \\
\hline Tecnologías RER & 1314 GWh & $1300 \mathrm{GWh}$ & $320 \mathrm{GWh}$ & $1400 \mathrm{GWh}$ \\
\hline $\begin{array}{c}\text { Centrales Hidroeléctricas } \leq \\
20 \mathrm{MW}\end{array}$ & $500 \mathrm{MW}$ & $681 \mathrm{GWh}$ & 1300 GWh & $450 \mathrm{GWh}$ \\
\hline
\end{tabular}

\begin{tabular}{|c|c|c|c|c|c|c|c|c|c|}
\hline \multirow{2}{*}{\multicolumn{2}{|c|}{$\begin{array}{c}\text { Precios Ofertados } \\
\text { US\$/MWh } \\
\text { Precio Mínimo }\end{array}$}} & \multicolumn{2}{|c|}{ 1ra Subasta } & \multicolumn{2}{|c|}{ 2da Subasta } & \multicolumn{2}{|c|}{ 3ra Subasta } & \multicolumn{2}{|c|}{ 4ta Subasta } \\
\hline & & \multirow{2}{*}{$\begin{array}{c}\begin{array}{c}\text { Precio } \\
\text { Máximo }\end{array} \\
65,52\end{array}$} & \multicolumn{2}{|c|}{ Precio Mínimo } & $\begin{array}{l}\text { Precio } \\
\text { Mínimo }\end{array}$ & $\begin{array}{l}\text { Precio } \\
\text { Máximo }\end{array}$ & Precio Mínimo & $\begin{array}{l}\text { Precio } \\
\text { Máximo }\end{array}$ & \\
\hline \multirow{3}{*}{$\begin{array}{l}\text { Tecnologías } \\
\text { RER }\end{array}$} & Eólica & & 87,0 & \multicolumn{2}{|c|}{69,0} & --- & --- & 36,84 & 37,83 \\
\hline & Solar & 215,0 & 225,0 & \multicolumn{2}{|c|}{119,90} & --- & --- & 47,98 & 48,5 \\
\hline & Biomasa & 52,0 & 110,0 & \multicolumn{2}{|c|}{99,99} & --- & --- & 77,0 & 77,0 \\
\hline \multicolumn{2}{|c|}{$\begin{array}{c}\text { Centrales Hidroeléctricas } \\
\leq 20 \mathrm{MW}\end{array}$} & 55,0 & 70,0 & 47,4 & 56,45 & 50,50 & 64,80 & 40,0 & 58,20 \\
\hline
\end{tabular}

5. Megavatio/hora. 
poder costear un proyecto de gran envergadura pasa primero por la determinación inicial de la estructura más conveniente.

El primer elemento a considerar es que este tipo de proyectos usualmente son desarrollados mediante un vehículo de propósito especial incorporado en el Perú para abocarse a su desarrollo, implementación y gestión de la Sociedad Vehículo Especial —denominada Social Purpose Vehicle, en adelante "SPV" por sus siglas en inglés-. Esto se debe, además de ciertos impactos tributarios, al hecho de que usualmente los accionistas o "sponsors" desean que los proyectos tengan sus propias alas, asuman sus responsabilidades de forma directa y que dentro de lo posible no dependan de forma exclusiva de los bolsillos de sus accionistas para lo cual recurren al financiamiento para obtener capital.

Considerando lo anterior llegamos entonces a la primera premisa, estos proyectos son usualmente financiados y no desarrollados exclusivamente con aportes de capital de sus socios. Los financistas, con la intención de no asumir de forma exclusiva los riesgos del proyecto, y a su vez para comprometer a los sponsors con este, normalmente desembolsan como máximo el $80 \%$ de los fondos estimados, debiendo ser la diferencia aportada por los accionistas.

Partiendo entonces de que los proyectos de este tipo son mayoritariamente financiados nace entonces la siguiente decisión, decidir entre las vías más idóneas de financiamiento, la "eficiencia financiera" se torna en las palabras claves a buscar. Es en esa disyuntiva que la estructura del RER empieza a tomar sentido para que sea el Project Finance aquella en la que se decante.

Recordemos así que bajo la regulación RER, el Estado se compromete, mediante un contrato de compra de energía, a adquirir por un plazo determinado y a un precio determinado la capacidad de generación de aquellas generadoras de energía renovable ganadoras del concurso. Esta suerte de apoyo se da ya bajo la lógica que no obstante la energía renovable tiende a ser más costosa que otras por diversos factores, y por ende acceder a contratos de venta de ener- gía con particulares puede ser más complejo, el Estado considera importante que esta sea parte de la matriz energética del país por sus beneficiosos vinculados mayoritariamente con el impacto ambiental.

Considerando entonces este respaldo estatal asegurando ingresos durante un plazo determinado, de cara a un financiamiento esto significa, en pocas palabras, un flujo mínimo predecible de ingresos, los cuales pueden servir para el repago de una deuda bajo un esquema de Project Finance.

¿En qué consiste el financiamiento mediante estructuras de Project Finance y por qué es tan popular su uso hoy? Se conoce bajo este nombre al mecanismo de financiación de proyectos que se sustenta en los flujos de caja positivos de este, entiéndase de la SPV, y no en la solvencia de los sponsors que se encuentran detrás del negocio y quienes son los beneficiarios del mismo.

En ese orden de ideas, en este tipo de financiación no importa tanto del valor de los activos que los sponsors puedan aportar como garantía o inclusive como capital, sino que prima la capacidad del proyecto en si para generar o levantar los recursos que le permitan (i) construir la infraestructura, (ii) mantener la operación y mantenimiento (iii) repagar las deudas; y (iv) repartir beneficios y dividendos.

Así, en este tipo de estructuras, la clave está en la efectiva asignación de riesgos. En el Project Finance, los riesgos se asignan de manera equitativa entre todas las partes que participan en la transacción, con el objetivo de conseguir eficiencias en la gestión y en la financiación. Así, el constructor, el operador, los accionistas, la SPV y por supuesto los financistas asumen los riesgos que le corresponden, vale decir, riesgos constructivos, riesgos de operación, riesgos financieros y otros.

La doctrina es muy unánime en señalar como rasgos distintivos de un Project Finance los siguientes, los cuales aplican a su vez para el financiamiento en Perú de plantas de energías renovables bajo el esquema RER:

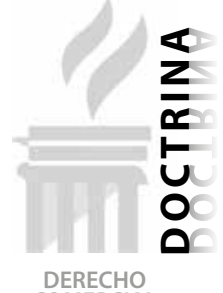


a) El tomador de la deuda, entiéndase la SPV, es una sociedad incorporada en el país por los sponsors únicamente para el desarrollaro del proyecto, la cual mantiene independencia financiera y jurídica de sus accionistas.

b) Normalmente los prestamistas reciben como única garantía el flujo de caja, las acciones de la SPV mediante garantías mobiliarias y los activos del proyecto mediante fideicomisos de activos, además de ser beneficiarios, de ser el caso, de las pólizas de seguros que sobre estas puedan haber.

Acá es importante remarcar que este es un escenario ideal para los desarrolladores quienes quieren alejar su patrimonio del de la SPV y sobre todo de los financistas. Sin embargo, hoy es muy usual que al menos en la etapa de construcción, cuando aún hay cierta incertidumbre sobre el flujo ya que la planta aun mantiene riesgos constructivos, los prestamistas soliciten garantías corporativas a los accionistas o desarrolladores para evitar asumir riesgos de que la planta tenga desperfectos en la etapa de construcción que eviten la generación de flujos para el repago.

c) Los flujos de efectivo generados deben cubrir los gastos operativos y de manutención de la planta y además poder cubrir los costos de la deuda financiera - capital e intereses-. En principio, solo los remanentes pueden ser repartidos como utilidades, ello luego de cubiertos estos y de cubierta cualquier garantía de repago.

d) Los plazos del financiamiento deben de calzar con los plazos de los contratos de venta de energía - en casos como el que aboca el presente- que suscriba el proyecto para asegurar que durante el plazo del financiamiento se cuente con ingresos claros suficientes para el repago.

e) Para el financiamiento de generadoras de energía renovable cuya producción es por definición no constante -energía eólica o energía solar, por ejemplo-y por ende su flujo de ingresos es en principio impredecible, los prestamistas mitigan el riesgo mediante proyección de ingresos sustentado en estadísticas.

\section{CONCLUSIONES Y APUNTES FINALES}

a) Los proyectos de energía renovables en el Perú han sido promovidos hasta la fecha por el Estado mediante licitaciones de contratos de suministro para la compra de energía.

b) El Project Finance es la estructura financiera más utilizada por los desarrolladores de proyectos de energías renovables utilizando esos contratos de suministro como base para el futuro repago de sus créditos.

c) En la medida que los costos de desarrollo de energías renovables disminuya, disminuirá también la necesidad de que el Estado, en la necesaria intención de promover energías renovables, se vea forzado a comprar dicha energía.

d) El mercado de desarrolladores y de prestamistas se ha acostumbrado a la existencia de estos contratos con el Estado para sostener los modelos financieros generándose una dependencia a un sistema cuya existencia debe podría desaparecer pronto.

e) Es fundamental que las empresas del sector eléctrico renovable desarrollen tecnología y eficiencias que permitan reducir y por qué no eliminar la dependencia a los subsidios. 